\title{
Detection of clinical progression through plasma ctDNA in metastatic melanoma patients: a comparison to radiological progression
}

\author{
Gabriela Marsavela ${ }^{1,2}$, Ashleigh C. McEvoy ${ }^{1}$, Michelle R. Pereira ${ }^{1}$, Anna L. Reid ${ }^{1,2}$, Zeyad Al-Ogaili ${ }^{3}$, Lydia Warburton ${ }^{1,2,4}$, \\ Muhammad A. Khattak ${ }^{1,5,6}$, Afaf Abed ${ }^{1,2}$, Tarek M. Meniawy ${ }^{1,4,5}$, Michael Millward ${ }^{1,4,5}$, Melanie R. Ziman ${ }^{1,7}$, Leslie Calapre ${ }^{1}$ and \\ Elin S. Gray (iD ${ }^{1,2}$ 凶
}

(c) The Author(s) 2021

BACKGROUND: The validity of circulating tumour DNA (ctDNA) as an indicator of disease progression compared to medical imaging in patients with metastatic melanoma requires detailed evaluation.

METHODS: Here, we carried out a retrospective ctDNA analysis of 108 plasma samples collected at the time of disease progression. We also analysed a validation cohort of 66 metastatic melanoma patients monitored prospectively after response to systemic therapy.

RESULTS: ctDNA was detected in $62 \%$ of patients at the time of disease progression. For 67 patients that responded to treatment, the mean ctDNA level at progressive disease was significantly higher than at the time of response $(P<0.0001)$. However, only 30 of these 67 (45\%) patients had a statistically significant increase in ctDNA by Poisson test. A validation cohort of 66 metastatic melanoma patients monitored prospectively indicated a 56\% detection rate of ctDNA at progression, with only two cases showing increased ctDNA prior to radiological progression. Finally, a correlation between ctDNA levels and metabolic tumour burden was only observed in treatment naïve patients but not at the time of progression in a subgroup of patients failing BRAF inhibition $(N=15)$.

CONCLUSIONS: These results highlight the low efficacy of ctDNA to detect disease progression in melanoma when compared mainly to standard positron emission tomography imaging.

British Journal of Cancer (2022) 126:401-408; https://doi.org/10.1038/s41416-021-01507-6

\section{INTRODUCTION}

Metastatic melanoma is the most aggressive type of skin cancer and is responsible for most skin cancer-related deaths [1, 2]. In the past decade, the clinical management of patients with advanced melanoma was revolutionised by the use of anti-programmed cell death 1 (PD1) and/or anti-cytotoxic-T-lymphocyte-associated antigen 4 (CTLA-4) immunotherapies, as well as BRAF/MEK inhibiting targeted therapies [3]. These treatments have significantly increased the 5-year survival rate of melanoma patients, which is currently at $52 \%$ for the nivolumab plus ipilimumab, $44 \%$ for nivolumab alone, $26 \%$ for ipilimumab and $34 \%$ for dabrafenib/trametinib $[4,5]$. Imaging scans, such as ${ }^{18} \mathrm{~F}$-labelled fluorodeoxyglucose positron emission tomography (FDG-PET) or computerised tomography (CT), are currently the clinical standard for treatment monitoring, but are costly and of limited accessibility in rural areas. Nonetheless, disease progression occurs in a large proportion of patients, even after treatment cessation [6]. Thus, biomarkers that can aid clinicians in accurately detecting progressive disease in patients are critical.
Circulating tumour DNA (ctDNA) is progressively used as a marker of disease status in multiple types of cancers, including melanoma [7]. This non-invasive real-time biomarker has been demonstrated to accurately reflect pretreatment tumour burden [8, 9], provide predictive or prognostic information and is a useful monitoring tool for treatment response [10-12]. However, very few studies [13-16] have defined the clinical validity of ctDNA to effectively identify disease progression in a large cohort of melanoma patients compared to medical imaging, the current gold standard for disease monitoring.

In this study, we quantified plasma ctDNA $(N=108)$ at the time of radiological disease progression in a cohort of metastatic melanoma patients treated with systemic therapy in the context of real-world clinical practice. We further evaluated the potential of ctDNA to detect disease progression in a prospective cohort of patients $(N=66)$. Finally, we evaluated the correlation between ctDNA levels and metabolic tumour burden (MTB) prior to treatment and at the time progression in a subgroup of patients treated with BRAF/MEK inhibitors $(N=15)$.

\footnotetext{
${ }^{1}$ School of Medical and Health Sciences, Edith Cowan University, Joondalup, WA, Australia. ${ }^{2}$ Centre for Precision Health, Edith Cowan University, Joondalup, WA, Australia. ${ }^{3}$ Department of Molecular Imaging and Therapy Service, Fiona Stanley Hospital, Murdoch, WA, Australia. ${ }^{4}$ Department of Medical Oncology, Sir Charles Gairdner Hospital, Nedlands, WA, Australia. ${ }^{5}$ School of Medicine, University of Western Australia, Crawley, WA, Australia. ${ }^{6}$ Department of Medical Oncology, Fiona Stanley Hospital, Murdoch, WA, Australia. ${ }^{7}$ School of Biomedical Science, University of Western Australia, Crawley, WA, Australia. ${ }^{\circledR}$ email: e.gray@ecu.edu.au
} 


\section{MATERIALS AND METHODS \\ Patients}

Metastatic melanoma patients undergoing systemic therapies were enrolled in the study between 2013 and 2019 at Sir Charles Gairdner Hospital (SCGH) and Fiona Stanley Hospital (FSH) in Perth, Western Australia. Written informed consent was obtained from all patients under approved Human Research Ethics Committee protocols from Edith Cowan University (Nos. 11543 and 18957) and SCGH (Nos. 2007-123 and 2013-246) in compliance with the Declaration of Helsinki. Experiments were performed according to institutional and national guidelines and regulations.

We retrospectively selected patients who had plasma samples collected close to the time of the first unequivocal radiological scan indicating disease progression (PD) after commencing therapy, i.e. either 1 week before or up to 4 weeks of PD, but without any change in treatment. Blood samples were collected in EDTA vacutainer or in Cell-Free DNA BCT ${ }^{\circledR}$ (Streck, La Vista, NE). Within $24 \mathrm{~h}$ of blood collection, plasma was separated by centrifugation at $300 \times g$ for $20 \mathrm{~min}$, followed by second centrifugation at $4700 \times g$ for $10 \mathrm{~min}$. Isolated plasma was stored at $-80^{\circ} \mathrm{C}$ until extractions were performed. A total of 108 plasma samples from 93 patients were included in this analysis. For 15 patients, samples collected at progression to first- and second-line treatment were included in the analysis. Plasma samples at the time of best overall clinical response to therapy were available for 62 of the 108 cases investigated and were compared with ctDNA levels at progressive disease.

For the prospective analysis, a total of 66 patients were enrolled and monitored for plasma ctDNA every 3-12 weeks, with a median follow-up duration of 66 weeks (range 26-110 weeks). All blood samples were collected in Cell-Free DNA BCT ${ }^{\oplus}$ (Streck) and plasma was stored at $-80^{\circ} \mathrm{C}$ until extractions were performed.

\section{ctDNA assessment}

Selection of mutational targets for ctDNA analysis was identified via standard pathology protocols or using a customised melanoma nextgeneration sequencing (NGS) panel (Illumina, San Diego, CA, USA) as described by Calapre et al. [17], based on the following criteria: (i) known melanoma hotspot mutation inBRAF, NRAS and/or TERT promoter; (ii) COSMIC/TCGA reported melanoma-associated mutations; (iii) other mutation with a PolyPhen score $>0.7$ and high variant allele frequency in the tumour. Commercially available and/or customised probes were used to analyse ctDNA by droplet digital PCR (ddPCR). A list of all mutations used for ctDNA detection is provided in Table S1. The limit of blank for each assay used for ctDNA detection was previously reported by Calapre et al. [17] and Marsavela et al. [18].

Cell-free DNA (cfDNA) was extracted from plasma using the QIAamp Circulating Nucleic Acid Kit (Qiagen, Hilden, Germany). Extracted cfDNA was then analysed by ddPCR as previously described [12]; samples that were negative in the first instance were tested further in duplicate to complete a triplicate set. A positive control, a healthy control and a notemplate control were included in each run. Only tests providing $>10,000$ droplets were used for analysis.

\section{Disease progression assessment}

Tumour disease progression was assessed radiologically by CT and/or FDGPET scans. Magnetic resonance imaging (MRI) of the brain was also used where indicated. Patients were considered to have PD if they developed new lesions, had a significant increase in tumour size as per RECIST 1.1 or iRECIST on CT, had increased metabolic activity on FDG-PET scan or presented a new or enlarging clinical lesion, confirmed by the treating clinician. Clinicians were blinded to the ctDNA result at the time of the scan.

\section{MTB analysis}

MTB was calculated from FDG-PET scans as described previously [8, 17]. All images were reviewed retrospectively by an experienced nuclear medicine physician (ZA-O) blinded to the ctDNA analysis. Total lesion glycolysis, which combines volumetric and metabolic information, was calculated for MTB evaluation $[19,20]$. Analyses were conducted on a Siemens Syngo via workstation (Siemens Healthcare GMbH, Erlangen, Germany).

\section{Statistics}

Differences between ctDNA levels were estimated by unpaired $t$ test from the log-transformed data. Differences between the detection rates were assessed using two-sided Fisher's exact test. Paired $t$ test was used to evaluate the difference between ctDNA levels at the time of response and clinical progression. All variables were tested for normality and equality variance. Statistical differences between ctDNA levels at the time of response and progression in the same individual were also assessed using a Poisson distribution test, using calculated mutant DNA concentrations and the number of DNA occupied droplet counts as analytical variables [21]. Correlations between MTB and ctDNA were carried using Pearson's correlation of the log-transformed values. Statistical analyses were performed using R version 5.2, GraphPad Prism version 5 and SPSSv22.0. Results were considered statistically significant at $P<0.05$.

\section{RESULTS}

\section{ctDNA detection at disease progression}

We retrospectively selected 108 plasma samples collected at the time of disease progression to determine the rate of ctDNA detection. Patient characteristics and clinical parameters for both cohorts are summarised in Table 1. The overall detection rate was $62 \%$ and was independent of the tumour BRAF mutational status (Fig. 1a). When subdivided by disease stage, patients with visceral metastases at progression (M1c and M1d with extracranial metastases) had higher ctDNA levels and detection rates compared to those patients who progressed with lymph node, subcutaneous or lung lesions (Fig. 1b). Only 9 of 19 cases with metastases in the skin, subcutaneous tissue or lymph node (M1a, $47 \%$ ) had detectable ctDNA at progression. In addition, only two of the five cases with lung metastases (M1b, 40\%) had detectable ctDNA. Notably, all but one of the nine patients with the intracranial disease only (M1d IC only) had undetectable ctDNA at the time of progression, in contrast to the high detection rate amongst those with extracranial involvement.

From a total of 108 cases, 67 (62\%) showed a response to therapy prior to progressive disease. Interestingly, patients who responded to treatment had significantly lower ctDNA levels at disease progression ( $P=0.046$; Fig. 2a). Moreover, the detection rate was significantly lower in patients who responded (35/67, $52 \%$ ) compared to those who did not respond to therapy and had no tumour size reduction $(32 / 41,78 \%, P=0.008)$.

In this subgroup of patients with samples taken at the time of response to treatment and PD, paired comparison of ctDNA levels showed a significant increase in ctDNA at progression relative to the point of response or nadir $(N=67, P<0.0001$; Fig. 2b). Of these, 22 cases (34\%) had undetectable ctDNA at the time of best response, which became positive at progression. However, only $30 / 67(45 \%)$ of all cases had a significant increase in ctDNA level when $\mathrm{ddPCR}$ results are compared using a Poisson test.

\section{ctDNA detection at the time of progression in a prospective cohort}

To validate these results, we prospectively recruited a total of 66 metastatic melanoma patients either commencing or undergoing systemic treatment, including two cases treated with adjuvant therapy after removal of isolated tumour metastases. Of the 64 patients with active disease, 14 did not respond to therapy and were excluded from the analysis. Of the 52 patients with objective responses $(N=50)$ or who had adjuvant therapy $(N=2), 23(44 \%)$ experienced progression during the follow-up period. However, for seven cases, we did not have a blood collection for ctDNA analysis within 2 weeks of disease progression. Therefore, followup collections for a total of 45 patients, 16 with PD and 29 with the ongoing response, were included in the final analysis presented in Fig. 3. The clinical characteristics of these 45 patients can be found in Table 1. The majority of patients were males $(101 / 153,66 \%)$ and treated with combination therapy, either dabrafenib/trametinib $(70 / 153,46 \%)$ or ipilimumab/nivolumab (30/153, 20\%). The prospective cohort largely consisted of patients who received immunotherapy and the proportion of BRAF mutants was lower than in the retrospective cohort. 
Table 1. Clinical characteristics at baseline of the melanoma samples included in the study.

\begin{tabular}{|c|c|c|}
\hline Variable & $\begin{array}{l}\text { Retrospective } \\
\text { cohort, } N=108(\%)\end{array}$ & $\begin{array}{l}\text { Prospective } \\
\text { cohort, } N=45(\%)\end{array}$ \\
\hline \multicolumn{3}{|l|}{ Age } \\
\hline$\leq 60$ & $54(50)$ & $24(53)$ \\
\hline$>60$ & $54(50)$ & $21(47)$ \\
\hline \multicolumn{3}{|l|}{ Gender } \\
\hline Female & $38(35)$ & $14(31)$ \\
\hline Male & $70(65)$ & $31(69)$ \\
\hline \multicolumn{3}{|l|}{ Mutation status } \\
\hline$B R A F$ mutant & $81(75)$ & $32(71)$ \\
\hline NRAS mutant & $11(10)$ & $4(9)$ \\
\hline BRAF/NRAS WT & $16(15)$ & $9(20)$ \\
\hline \multicolumn{3}{|l|}{ Treatment } \\
\hline \multicolumn{3}{|l|}{$\mathrm{ICI}$} \\
\hline Pembrolizumab & $22(20)$ & $6(13)$ \\
\hline Ipilimumab/Nivolumab & $15(14)$ & $15(34)$ \\
\hline Ipilimumab & $11(10)$ & \\
\hline \multicolumn{3}{|l|}{ Targeted therapies } \\
\hline Vemurafenib & $3(3)$ & $1(2)$ \\
\hline Dabrafenib/Trametinib & $54(50)$ & $16(36)$ \\
\hline $\begin{array}{l}\text { Vemurafenib/ } \\
\text { Cobimetinib }\end{array}$ & $2(2)$ & $4(9)$ \\
\hline \multicolumn{3}{|l|}{ Adjuvant } \\
\hline Nivolumab & $1(1)$ & $1(2)$ \\
\hline Clinical trials & & $2(4)$ \\
\hline No progressive disease & - & $29(64)$ \\
\hline Progressive disease & $108(100)$ & $16(36)$ \\
\hline \multicolumn{3}{|l|}{ Response prior to PD } \\
\hline Yes & $67(62)$ & $16(36)$ \\
\hline $\begin{array}{l}\text { ctDNA not } \\
\text { elevated at PD }\end{array}$ & $34(31)$ & $7(16)$ \\
\hline $\begin{array}{l}\text { ctDNA } \\
\text { elevated at PD }\end{array}$ & $33(31)$ & $9(20)$ \\
\hline $\begin{array}{l}\text { ctDNA statistically } \\
\text { elevated at PD }\end{array}$ & $20(19)$ & $5(11)$ \\
\hline No & $41(38)$ & - \\
\hline $\begin{array}{l}\text { AJCC stage/M } \\
\text { classification at PD }\end{array}$ & & $N=16$ \\
\hline M1a & $19(18)$ & $5(31)$ \\
\hline M1b & $5(5)$ & $2(13)$ \\
\hline M1c & $51(47)$ & $4(25)$ \\
\hline M1d & $33(30)$ & $5(31)$ \\
\hline \multicolumn{3}{|l|}{ Brain-only metastasis at PD } \\
\hline Yes & $9(8)$ & $4(25)$ \\
\hline No & $99(92)$ & $12(75)$ \\
\hline
\end{tabular}

$\mathrm{ICI}$ immune checkpoint inhibitors, PD progressive disease.

Most patients experiencing disease progression were treated with BRAF/MEK inhibitors $(11 / 16,69 \%$, Fig. 3). Amongst the 16 patients who relapsed, disease progression was detected at a median time of 31 weeks from the commencement of treatment (range 10-64 weeks). Only 9 of 16 patients had elevated ctDNA levels at the time of progression (56\%; Fig. 3). From the nine patients with increased ctDNA levels at disease progression, except for case \#1069, all others had a significant increase in ctDNA level when ddPCR results were compared using Poisson statistics. By contrast, seven cases remained ctDNA negative despite radiological disease progression (44\%). Of these, three had intracranial disease-only progression (red asterisks; Fig. 3). Of note, one of the three patients with the intracranial disease only (\#1069) developed a leptomeningeal disease and had a detectable but low plasma ctDNA (1.4 copies $/ \mathrm{mL}$ ) at progression.

Notably, four patients had no detectable plasma ctDNA at the time of progression with clear extracranial disease. One patient showed progression in the right external iliac node (\#693), one had a new subcutaneous lesion on the left thigh (\#1093), another developed several new bilateral pulmonary nodules at the time of progression (\#806) and one (\#1108) showed bone recurrence at the $\mathrm{T} 2$ vertebral body.

For two cases ctDNA became detectable 10-25 weeks prior to radiological disease progression (Fig. 3). Patient \#974 showed partial response prior to progressive disease in lung and lymph nodes detected by PET scans. Analysis of plasma collected 24 weeks prior to progression indicated an increase in ctDNA levels, but radiological examination demonstrated no evidence of progressive disease. Similarly, patient \#825 showed increasing ctDNA levels at $\sim 13$ weeks before the clinical and radiological progressive disease was identified in multiple lymph nodes, gallbladder, duodenum and subcutaneous nodules.

Patients without unequivocal disease progression $(N=29)$ were also followed up for a median of 66 weeks (range 26-110). Plasma ctDNA was undetectable in the majority of plasma samples. However, we observed that ten patients (34\%) had intermittent low ctDNA levels without apparent clinical cause. Of note, ctDNA levels were always below 10 copies $/ \mathrm{mL}$ and undetectable in later follow-up samples.

\section{Correlation between ctDNA levels versus MTB at baseline and disease progression}

Previously, we observed that in pretreatment samples from metastatic melanoma patients, ctDNA plasma concentrations strongly correlated with MTB derived from PET scans [8]. Here, we investigated the correlation between ctDNA and MTB at the time of progression in 15 patients treated with dabrafenib plus trametinib with matching samples at baseline and progression. We selected this subgroup as they constitute the majority of patients monitored in this study who developed disease progression after an objective response during the ctDNA monitoring period.

While we found a strong correlation between ctDNA and MTB at baseline $(r=0.8659, P<0.0001)$, no correlation was apparent at disease progression $(r=0.4923, P>0.05)$ (Fig. 4). These results suggest that ctDNA levels do not reflect disease burden and its metabolic activity at progression.

\section{DISCUSSION}

The high number of therapies available for late-stage melanoma necessitates an efficient stratification of patients to appropriate treatments. In addition, rigorous monitoring for timely detection of disease progression is also necessary, to ensure swift modification of treatment and improve patient outcomes. Plasma ctDNA has been heralded as an alternative pathway to monitor disease in cancer patients, and can potentially provide lead time to overt progression within which treatment could be modified [13]. Here, we made use of a real-world cohort of metastatic melanoma patients to determine the reliability of ctDNA for the detection of disease progression. Our results demonstrated that ctDNA elevation was only able to indicate the progression in $52-56 \%$ of patients, highlighting the limitations of ctDNA as a reliable marker to monitor progression alone.

We observe that low ctDNA copies ( $<10$ copies $/ \mathrm{mL}$ of plasma) were detected sporadically in patients who do not show radiological progression. Therefore, stringent criteria are required to indicate disease progression by ctDNA detection. We attempted to address this by conducting a Poisson test to 

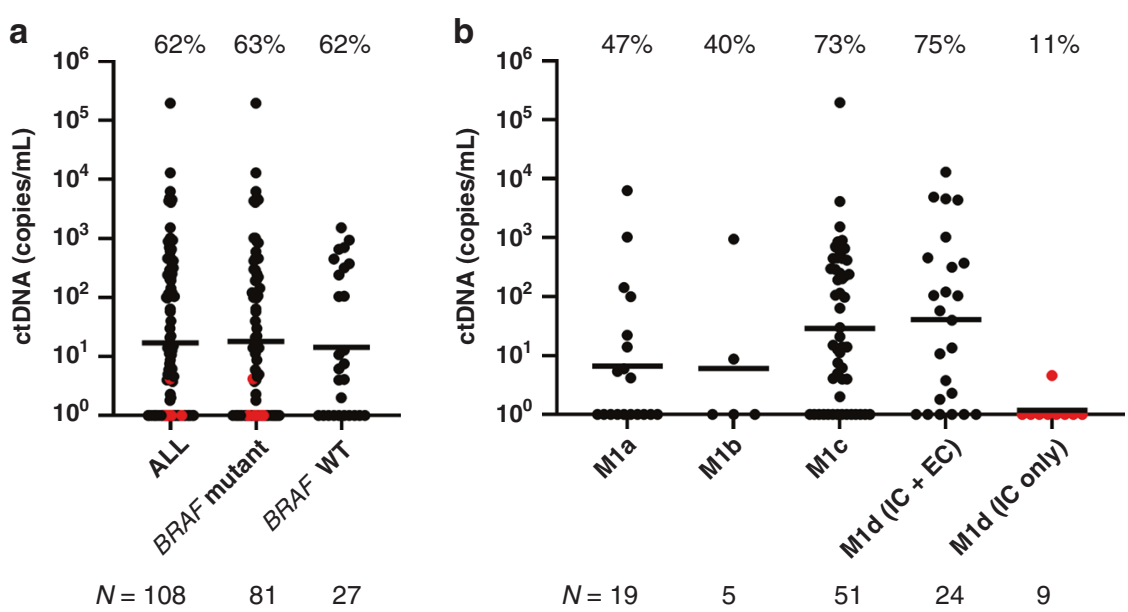

Fig. 1 ctDNA quantification in melanoma patients at the time of progressive disease on systemic therapy. a Plasma ctDNA levels (copies/ $\mathrm{mL}$ of plasma) in melanoma samples $(N=108)$, stratified by mutation status. Red dots identify patients with the intracranial disease only. $\mathbf{b}$ M1d cases were further subdivided into those with extracranial (IC + EC) and those with brain-only metastases (IC only). Percentages denote the frequency of patients with detectable ctDNA. The geometric mean of ctDNA concentrations is indicated for each group by a line.

$$
\underset{52 \%}{P\left(X^{2} \text { test }\right)=}=\underset{78 \%}{0.008}
$$

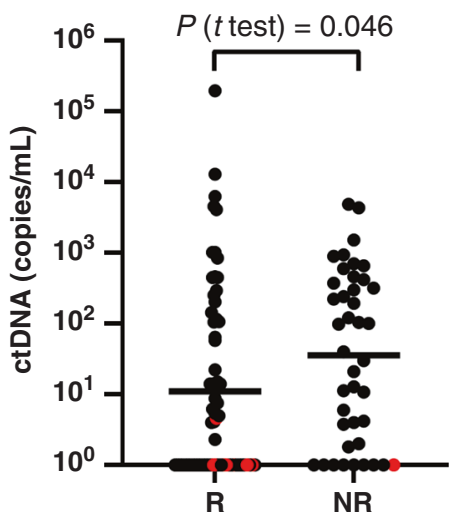

$N=67$

41 b

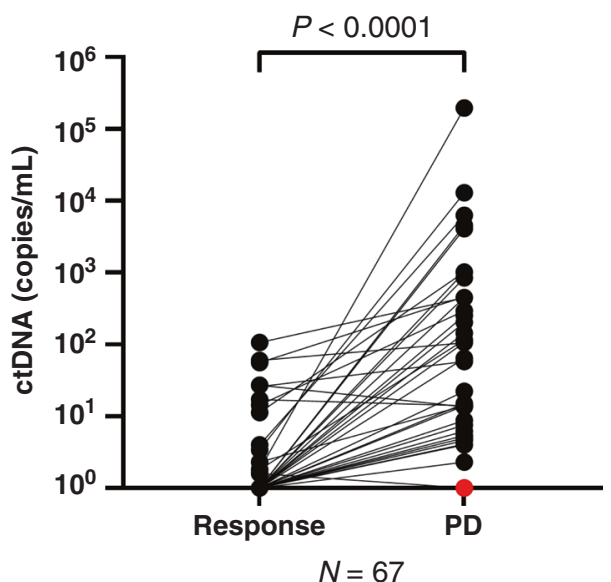

Fig. 2 Plasma ctDNA levels are relative to progression. a Plasma ctDNA levels (copies $/ \mathrm{mL}$ of plasma) in melanoma samples at the time of progression in patients who had a response to therapy (R) or did not show response to treatment (NR). The geometric mean of ctDNA concentrations is indicated for each group by a line. The $P$ values of a $t$ test comparing the log-transformed ctDNA levels and Fisher's exact test comparing detection rates are indicated above each comparison. $\mathbf{b}$ Changes in ctDNA levels between the time of response and the time of progression. Percentages denote the frequency of patients with detectable ctDNA. Red dots identify patients with intracranial disease only. The geometric mean of ctDNA concentrations is indicated for each group by a line. Paired $t$ test $P$ value of the log-transformed ctDNA levels is indicated above the graph.

determine statistically significant increases in ctDNA. Accordingly, only 30 of 67 (45\%, retrospective cohort) and 8 of 9 (89\%, prospective cohort) patients were found to have a statistically significant increase in ctDNA concentration at PD relative to the response. Overall, this reduces the rate of ctDNA detection to $47 \%$. These results highlight the low efficacy of ctDNA to detect PD in most patients when compared to standard PET imaging.

Notably, one major challenge for the use of ctDNA as a monitoring biomarker of progressive disease is the lack of detectable tumour-derived fragments in the plasma of patients with brain metastases $[22,23]$. In our study, 3 of the $7(43 \%)$ ctDNA-negative patients in the prospective study and 8 out of 41 (20\%) of ctDNA-negative cases in the retrospective study had intracranial disease only at the time of progression. Accounting for patients who had an initial response to therapy in both the retrospective and prospective cohort, a significant proportion had intracranial disease progression (12-19\%). The presence of isolated brain metastases poses a significant limitation for the use of plasma ctDNA as a surveillance biomarker, considering that at the time of diagnosis of metastatic melanoma nearly $20 \%$ of patients will only have metastasis in the brain and $>50 \%$ of patients undergoing targeted therapies or immunotherapies will eventually develop the progressive disease in the brain [24-26]. Thus, for these patients, plasma ctDNA is not suitable for monitoring disease progression, and MRI may remain a critical modality for monitoring disease progression in melanoma. Nonetheless, previous research [27-30] has shown that ctDNA is detectable in the cerebrospinal fluid (CSF) of cancer patients with brain metastases only. Assessment of the dynamic changes of ctDNA in CSF, but not in plasma, is potentially a valid surrogate marker in such situations. 

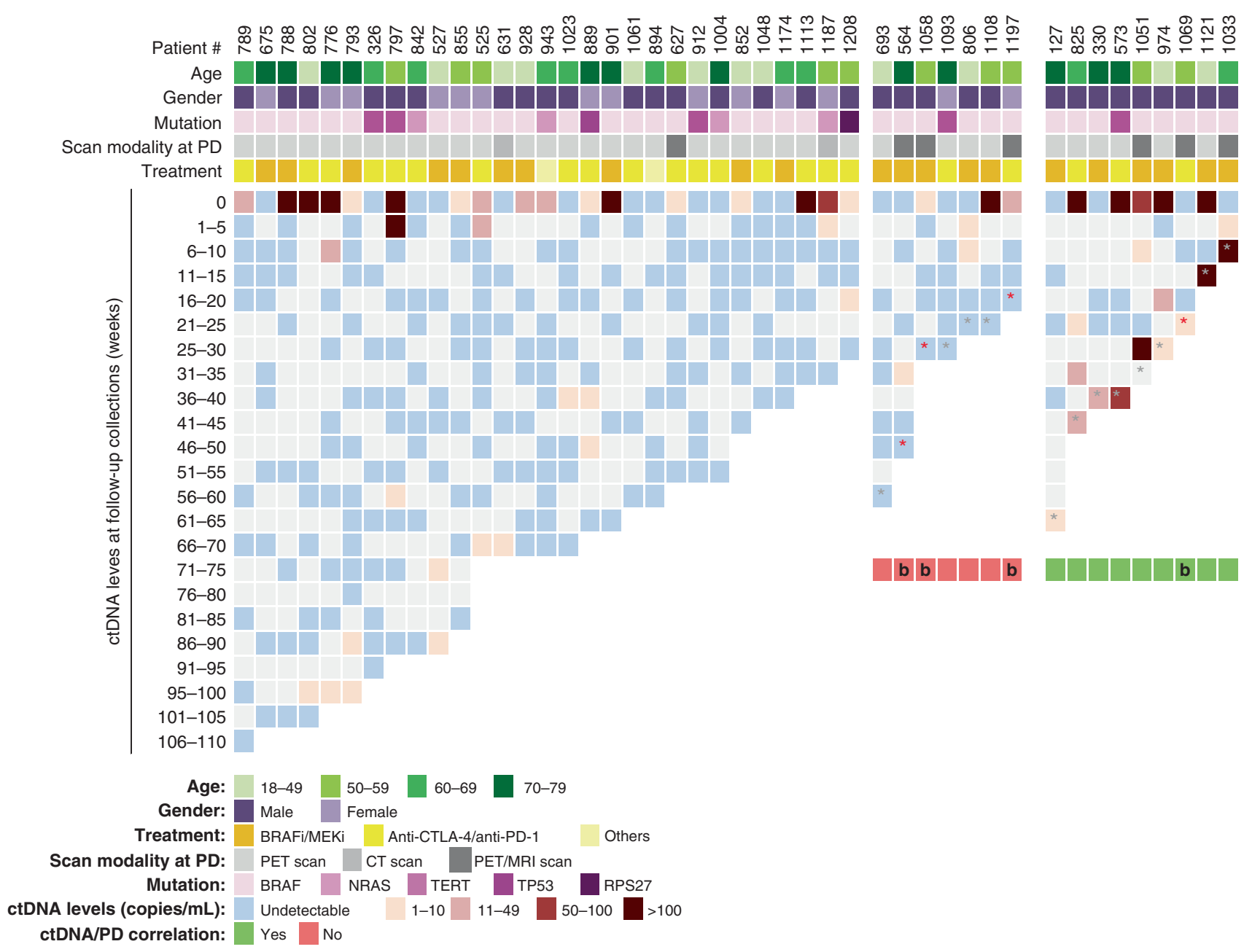

Age
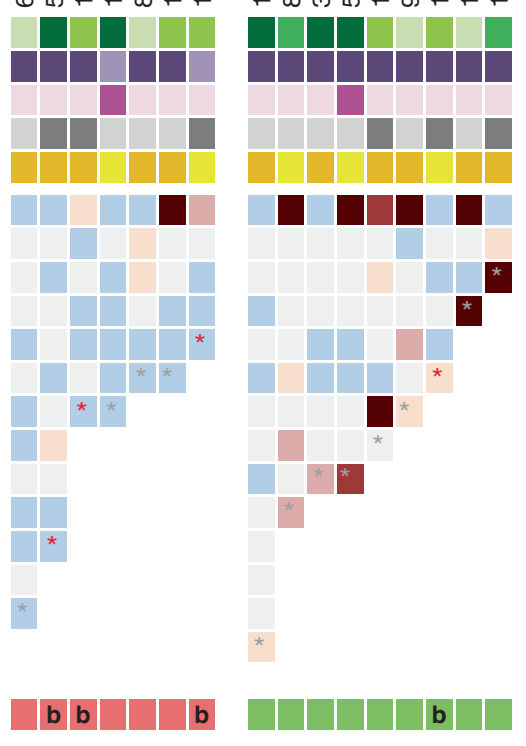

Fig. 3 Plasma ctDNA levels in the prospective cohort during the follow-up period relative to clinical progression. Columns represent each patient's age, gender, mutation status, scan modality used for monitoring, treatment and longitudinal quantitative ctDNA results. Columns are stratified into patients without or with disease progression, and the latter separated into those with detectable or undetectable ctDNA. bIntracranial disease only. Grey*- Progressive disease. Red *- Presence of only intracranial malignant disease at progressive disease.
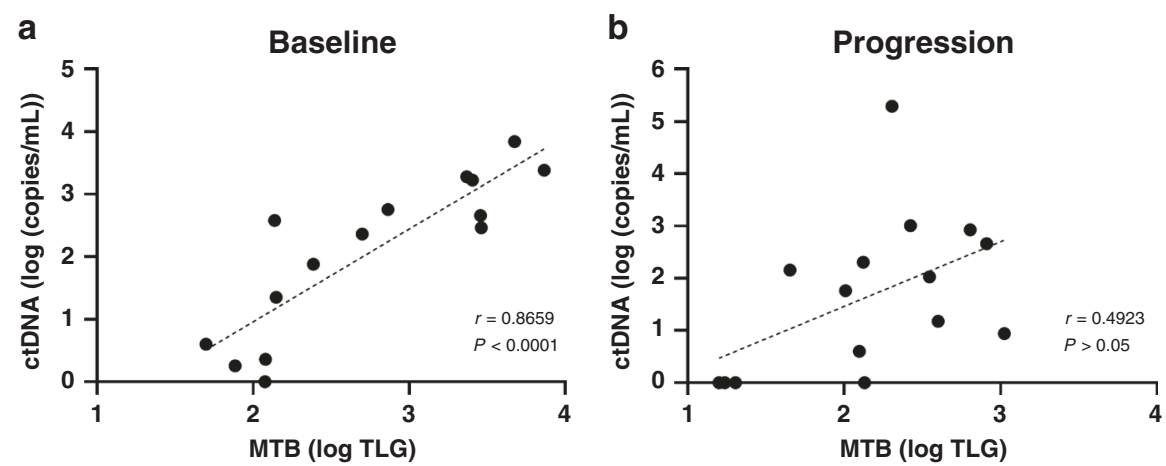

Fig. 4 Plasma ctDNA and MTB correlation. a, b Correlation between ctDNA levels and MTB in melanoma patients treated with combination BRAF/MEKi-targeted therapies at baseline (a) and radiological detection of progression (b). Pearson's correlation coefficient and $P$ values are indicated for each comparison.

Immunotherapy with anti-CTLA-4 or anti-PD-1 agents presents a challenge for oncologists due to pseudoprogression. This pattern is defined as initial radiographic tumour growth followed by regression and occurs in $10-15 \%$ of patients with metastatic disease [31, 32]. This delayed immune response could lead to an erroneous indication of refractory disease, which is associated with ctDNA negativity [33]. In this study, disease progression by radiological scans were all confirmed by a second scan that shows disease progression or a tumour biopsy that confirms malignancy. Based on these parameters, the lack of ctDNA at progression was therefore not attributable to potential pseudoprogression events.

Despite the strong suggestions that ctDNA may provide a lead time for detection of disease progression [9, 12-14], here we 
observed that ctDNA failed to successfully detect disease progression in patients that initially responded to therapy. Only a few studies have previously assessed the ctDNA detection rate at disease progression in melanoma. Rowe et al. [14] presented a detection rate of $100 \%$ in a small cohort of five patients that developed disease progression after showing response to therapy. Moreover, they reported detectable ctDNA in four of these patients prior to radiological detection of progression. However, the statistical significance of this increase is not described. Similarly, Haselmann et al. [13] has also reported a high percentage of cases with ctDNA detection prior to radiological progression $(61 \%, N=11 / 18)$. However, the prior response to therapy in these patients is unclear. In addition, from the 18 cases monitored, 14 cases had detectable ctDNA at disease progression (78\%). More recently, Váraljai et al. described that ctDNA increase preceded radiologic progression with an average lead time window of 3.5 months in $86 \%$ of the 36 melanoma patients [34]. A study by Schreuer et al. described an increase in BRAF mutant ctDNA in patients treated with targeted therapies at the time of radiological disease progression assessed by PET scans in 36 patients. This increase was detected with a sensitivity of $70 \%$ $(N=19 / 27)$ and specificity of $100 \%$ [16]. Based on our results and that of others, there is currently no congruence of ctDNA detection rate at PD in melanoma, and therefore further studies are required to elucidate factors influencing these results.

The lower detection rate reported in our study, in contrast to the above-mentioned reports, may be influenced by the imaging screening modality. For most of our patients (66\%), disease progression was identified by PET scans. The widespread use of PET for monitoring melanoma patients in Australia results in more sensitive surveillance of progression compared to CT scans. In a meta-analysis that pooled data from 74 studies containing 10,528 melanoma patients, PET was found to be superior to CT for the surveillance of metastatic disease [35]. This PET improved performance in depicting metastatic lesions over conventional imaging modalities; however, CT has been commonly described in the literature $[36,37]$.

Reconciling the findings of previous studies that used CT scan as a mode of restaging [13, 14, 34], and with our observation predominantly contrasted to PET scans, ctDNA detection rates at progression is likely to have comparable if not superior sensitivity when compared only to CT scans, given that there must be a sizable volume of disease for a significant increase to be detected. Thus, the clinical utility of ctDNA for progressive disease monitoring should be evaluated relative to the prevalent imaging modality. Despite ctDNA not showing high detection rates when compared with PET monitoring, its use may allow monitoring of patients that live in rural areas, potentially aiding adjustment of inequality of health access.

Previously, we have shown that patients with $<10$ MTB score, which equates to a low disease burden, have ctDNA levels that fall within the range of sampling error and therefore affect detection. In addition, despite the high correlation found previously between ctDNA levels and disease burden [8], we observed that ctDNA levels do not correlate with MTB at the time of progression in patients treated with targeted therapies. This also aligns with our previous report that ctDNA is not predictive of outcome to secondline immunotherapy following targeted therapy failure [18].

It is possible that cellular mechanisms that mediate treatment resistance may also interfere with cell apoptosis rates and ctDNA shedding. The cellular mechanism through which ctDNA is shed is poorly understood and its source has been extended to apoptotic tumour cells [38], tumour-derived extracellular vesicles [39], disseminated tumour cells and circulating tumour cells [40, 41]. However, no systematic study is yet to discover whether location within an organ, vascularisation or mitotic rate affect the amount of released ctDNA [42]. In addition, Smith et al. [43] have shown that the number of macrophages and tumour necrosis factor-a expression within the tumour is increased in patients treated with BRAF and MEK inhibitors. The increased presence of macrophages in relapsed tumours resistant to MAPK inhibitors might play an important role in clearing apoptotic cells and maintaining low ctDNA levels in the blood. Improved methods for the detection of ctDNA need to be accompanied by an improved understanding of the relationship between ctDNA release and the pathological state of the tumour.

There are a few limitations in our retrospective study. The timing of the blood collections relative to imaging scans varied between patients. Given the short half-life of ctDNA, blood drawn should ideally be conducted immediately prior to imaging to ensure that the ctDNA detected is a true representation of the lesions identified by the imaging technique. Moreover, preanalytical factors such as plasma separation time, centrifugation speed or plasma extraction volume may also have affected our results [44].

The targeted principle of the ddPCR assay may also be a limiting factor in the detection rate of ctDNA at progression. Studies exploring the use of untargeted methods that simultaneously interrogate multiple cancer-specific variants based on the use of NGS techniques have also been shown to improve both sensitivity and specificity of ctDNA detection $[45,46]$. As sequencing costs are reduced, NGS-based untargeted strategies may become clinically feasible.

In summary, our real-world cohort study highlights the urgent need to improve the methods used to detect ctDNA at disease progression and investigate the biological nature of ctDNA shedding to increase the clinical application of this non-invasive liquid biopsy. Future clinical trials with simultaneous imaging evaluations and ctDNA plasma sampling are needed to accurately define the clinical utility of ctDNA at detecting disease progression.

\section{DATA AVAILABILITY}

The datasets generated during the current study are available from the corresponding author on reasonable request.

\section{REFERENCES}

1. Ferlay J, Soerjomataram I, Dikshit R, Eser S, Mathers C, Rebelo M, et al. Cancer incidence and mortality worldwide: sources, methods and major patterns in GLOBOCAN 2012. Int J Cancer. 2015;136:E359-86.

2. Bray F, Ferlay J, Soerjomataram I, Siegel RL, Torre LA, Jemal A. Global cancer statistics 2018: GLOBOCAN estimates of incidence and mortality worldwide for 36 cancers in 185 countries. Cancer J Clin. 2018;68:394-424.

3. Luke JJ, Flaherty KT, Ribas A, Long GV. Targeted agents and immunotherapies: optimizing outcomes in melanoma. Nat Rev Clin Oncol. 2017;14:463-82.

4. Robert C, Grob JJ, Stroyakovskiy D, Karaszewska B, Hauschild A, Levchenko E, et al. Five-year outcomes with dabrafenib plus trametinib in metastatic melanoma. N Engl J Med. 2019;381:626-36.

5. Larkin J, Chiarion-Sileni V, Gonzalez R, Grob J-J, Rutkowski P, Lao CD, et al. Fiveyear survival with combined nivolumab and ipilimumab in advanced melanoma. N Engl J Med. 2019;381:1535-46.

6. Patrinely JR Jr., Baker LX, Davis EJ, Song H, Ye F, Johnson DB. Outcomes after progression of disease with anti-PD-1/PD-L1 therapy for patients with advanced melanoma. Cancer. 2020;126:3448-55.

7. Siravegna G, Mussolin B, Venesio T, Marsoni S, Seoane J, Dive C, et al. How liquid biopsies can change clinical practice in oncology. Ann Oncol. 2019;30:1580-90.

8. McEvoy AC, Warburton L, Al-Ogaili Z, Celliers L, Calapre L, Pereira MR, et al. Correlation between circulating tumour DNA and metabolic tumour burden in metastatic melanoma patients. BMC Cancer. 2018;18:726.

9. Wong SQ, Raleigh JM, Callahan J, Vergara IA, Ftouni S, Hatzimihalis A, et al. Circulating tumor DNA analysis and functional imaging provide complementary approaches for comprehensive disease monitoring in metastatic melanoma. JCO Precis Oncol. 2017;1:1-14.

10. Ascierto PA, Minor D, Ribas A, Lebbe C, O'Hagan A, Arya N, et al. Phase II trial (BREAK-2) of the BRAF inhibitor dabrafenib (GSK2118436) in patients with metastatic melanoma. J Clin Oncol. 2013;31:3205-11. 
11. Santiago-Walker A, Gagnon R, Mazumdar J, Casey M, Long GV, Schadendorf D, et al. Correlation of BRAF mutation status in circulating-free DNA and tumor and association with clinical outcome across four BRAFi and MEKi clinical trials. Clin. Cancer Res. 2016;22:567-74.

12. Gray ES, Rizos H, Reid AL, Boyd SC, Pereira MR, Lo J, et al. Circulating tumor DNA to monitor treatment response and detect acquired resistance in patients with metastatic melanoma. Oncotarget. 2015;6:42008-18.

13. Haselmann V, Gebhardt C, Brechtel I, Duda A, Czerwinski C, Sucker A, et al. Liquid profiling of circulating tumor DNA in plasma of melanoma patients for companion diagnostics and monitoring of BRAF inhibitor therapy. Clin Chem. 2018;64:830-42.

14. Rowe SP, Luber B, Makell M, Brothers $P$, Santmyer J, Schollenberger MD, et al. From validity to clinical utility: the influence of circulating tumor DNA on melanoma patient management in a real-world setting. Mol Oncol. 2018;12:1661-72.

15. Seremet T, Jansen Y, Planken S, Njimi H, Delaunoy M, El Housni H, et al. Undetectable circulating tumor DNA (ctDNA) levels correlate with favorable outcome in metastatic melanoma patients treated with anti-PD1 therapy. J Transl Med. 2019;17:303.

16. Schreuer M, Meersseman G, Van Den Herrewegen S, Jansen $Y$, Chevolet I, Bott A, et al. Quantitative assessment of BRAF V600 mutant circulating cell-free tumor DNA as a tool for therapeutic monitoring in metastatic melanoma patients treated with BRAF/MEK inhibitors. J Transl Med. 2016;14:95.

17. Calapre L, Giardina T, Robinson C, Reid AL, Al-Ogaili Z, Pereira MR, et al. Locusspecific concordance of genomic alterations between tissue and plasma circulating tumor DNA in metastatic melanoma. Mol Oncol. 2019;13:171-84.

18. Marsavela G, Lee J, Calapre L, Wong SQ, Pereira MR, McEvoy AC, et al. Circulating Tumor DNA Predicts Outcome from First-, but not Second-line Treatment and Identifies Melanoma Patients Who May Benefit from Combination Immunotherapy. Clin Cancer Res. 2020;26:5926-33. https://doi.org/10.1158/1078-0432.CCR20-2251.

19. Chen HH, Chiu NT, Su WC, Guo HR, Lee BF. Prognostic value of whole-body total lesion glycolysis at pretreatment FDG PET/CT in non-small cell lung cancer. Radiology. 2012;264:559-66.

20. Kim TM, Paeng JC, Chun IK, Keam B, Jeon YK, Lee SH, et al. Total lesion glycolysis in positron emission tomography is a better predictor of outcome than the International Prognostic Index for patients with diffuse large B cell lymphoma. Cancer. 2013;119:1195-202.

21. Gu K, Ng HKT, Tang ML, Schucany WR. Testing the ratio of two Poisson rates. Biometrical J. 2008;50:283-98.

22. Lee JH, Menzies AM, Carlino MS, McEvoy AC, Sandhu S, Weppler AM, et al. Longitudinal Monitoring of ctDNA in Patients with Melanoma and Brain Metastases Treated with Immune Checkpoint Inhibitors. Clin Cancer Res. 2020;26:406471. https://doi.org/10.1158/1078-0432.CCR-19-3926.

23. Bettegowda C, Sausen M, Leary RJ, Kinde I, Wang Y, Agrawal N, et al. Detection of circulating tumor DNA in early- and late-stage human malignancies. Sci Transl Med. 2014;6:224ra224.

24. Spagnolo F, Picasso V, Lambertini M, Ottaviano V, Dozin B, Queirolo P. Survival of patients with metastatic melanoma and brain metastases in the era of MAPkinase inhibitors and immunologic checkpoint blockade antibodies: a systematic review. Cancer Treat Rev. 2016;45:38-45.

25. Davies MA, Liu P, Mclntyre S, Kim KB, Papadopoulos N, Hwu WJ, et al. Prognostic factors for survival in melanoma patients with brain metastases. Cancer. 2011;117:1687-96.

26. Bafaloukos D, Gogas $H$. The treatment of brain metastases in melanoma patients Cancer Treat Rev. 2004:30:515-20.

27. Ma C, Yang X, Xing W, Yu H, Si T, Guo Z. Detection of circulating tumor DNA from non-small cell lung cancer brain metastasis in cerebrospinal fluid samples. Thorac Cancer. 2020;11:588-93.

28. Momtaz P, Pentsova E, Abdel-Wahab O, Diamond E, Hyman D, Merghoub T, et al. Quantification of tumor-derived cell free DNA(cfDNA) by digital PCR (DigPCR) in cerebrospinal fluid of patients with BRAFV600 mutated malignancies. Oncotarget. 2016;7:85430-6.

29. De Mattos-Arruda L, Mayor R, Ng CK, Weigelt B, Martinez-Ricarte F, Torrejon D, et al. Cerebrospinal fluid-derived circulating tumour DNA better represents the genomic alterations of brain tumours than plasma. Nat Commun. 2015;6:8839.

30. Ballester LY, Glitza Oliva IC, Douse DY, Chen MM, Lan C, Haydu LE, et al. Evaluating circulating tumor DNA from the cerebrospinal fluid of patients with melanoma and leptomeningeal disease. J Neuropathol Exp Neurol. 2018;77:628-35.

31. Long GV, Weber JS, Larkin J, Atkinson V, Grob J-J, Schadendorf D, et al. Nivolumab for patients with advanced melanoma treated beyond progression: analysis of 2 phase 3 clinical trials. JAMA Oncol. 2017;3:1511-9.

32. Wolchok JD, Hoos A, O'Day S, Weber JS, Hamid O, Lebbé C, et al. Guidelines for the evaluation of immune therapy activity in solid tumors: immune-related response criteria. Clin. Cancer Res. 2009;15:7412-20.
33. Lee JH, Long GV, Menzies AM, Lo S, Guminski A, Whitbourne K, et al. Association between circulating tumor DNA and pseudoprogression in patients with metastatic melanoma treated with anti-programmed cell death 1 antibodies. JAMA Oncol. 2018:4:717-21.

34. Váraljai R, Wistuba-Hamprecht K, Seremet T, Diaz JMS, Nsengimana J, Sucker A, et al. Application of circulating cell-free tumor DNA profiles for therapeutic monitoring and outcome prediction in genetically heterogeneous metastatic melanoma. JCO Precis Oncol. 2019;3:1-10.

35. Xing $\mathrm{Y}$, Bronstein $\mathrm{Y}$, Ross MI, Askew RL, Lee JE, Gershenwald JE, et al. Contemporary diagnostic imaging modalities for the staging and surveillance of melanoma patients: a meta-analysis. J. Natl Cancer Inst. 2011;103:129-42.

36. Perng P, Marcus C, Subramaniam RM. 18F-FDG PET/CT and melanoma: staging, immune modulation and mutation-targeted therapy assessment, and prognosis. Am J Roentgenol. 2015;205:259-70.

37. Reinhardt MJ, Joe AY, Jaeger U, Huber A, Matthies A, Bucerius J, et al. Diagnostic performance of whole body dual modality 18F-FDG PET/CT imaging for $\mathrm{N}$ - and $\mathrm{M}$-staging of malignant melanoma: experience with 250 consecutive patients. J Clin Oncol. 2006;24:1178-87.

38. Jahr S, Hentze H, Englisch S, Hardt D, Fackelmayer FO, Hesch RD, et al. DNA fragments in the blood plasma of cancer patients: quantitations and evidence for their origin from apoptotic and necrotic cells. Cancer Res. 2001;61: 1659-65.

39. Thakur BK, Zhang H, Becker A, Matei I, Huang Y, Costa-Silva B, et al. Doublestranded DNA in exosomes: a novel biomarker in cancer detection. Cell Res. 2014;24:766-9.

40. Stephenson D, Nahm C, Chua T, Gill A, Mittal A, de Reuver $P$, et al. Circulating and disseminated tumor cells in pancreatic cancer and their role in patient prognosis: a systematic review and meta-analysis. Oncotarget. 2017;8:107223-36.

41. Aya-Bonilla C, Gray ES, Manikandan J, Freeman JB, Zaenker P, Reid AL, et al. Immunomagnetic-Enriched Subpopulations of Melanoma Circulating Tumour Cells (CTCs) Exhibit Distinct Transcriptome Profiles. Cancers (Basel). 2019;11:157. https://doi.org/10.3390/cancers11020157.

42. Papadopoulos N. Pathophysiology of ctDNA Release into the Circulation and Its Characteristics: What Is Important for Clinical Applications. Recent Results Cancer Res. 2020;215:163-80.

43. Smith MP, Sanchez-Laorden B, O'Brien K, Brunton H, Ferguson J, Young H, et al The immune microenvironment confers resistance to MAPK pathway inhibitors through macrophage-derived TNFa. Cancer Discov. 2014;4:1214-29.

44. Gerber T, Taschner-Mandl S, Saloberger-Sindhöringer L, Popitsch N, Heitzer E, Witt $\mathrm{V}$, et al. Assessment of pre-analytical sample handling conditions for comprehensive liquid biopsy analysis. J Mol Diagn. 2020;22:1070-86.

45. Wan JCM, Heider K, Gale D, Murphy S, Fisher E, Mouliere F, et al. ctDNA monitoring using patient-specific sequencing and integration of variant reads. Sci Transl Med. 2020;12:eaaz8084.

46. McDonald BR, Contente-Cuomo T, Sammut SJ, Odenheimer-Bergman A, Ernst B, Perdigones $\mathrm{N}$, et al. Personalized circulating tumor DNA analysis to detect residual disease after neoadjuvant therapy in breast cancer. Sci Transl Med. 2019;11: eaax7392. https://doi.org/10.1126/scitransImed.aax7392.

\section{ACKNOWLEDGEMENTS}

We thank the melanoma patients for their support and participation in the study. We also thank Paula van Miert, Mike Morici, Aaron Beasley, Danielle Bartlett and Pauline Zaenker for their help in the collection and processing of blood samples from patients and healthy controls. Furthermore, we extend our thanks to Dr. Benhur Amanuel, Dr. Cleo Robinson and Dr. Tindaro Giardina from PathWest for assistance with the mutation profiling of tumours.

\section{AUTHOR CONTRIBUTIONS}

GM performed experiments, edited the manuscript, realised data curation, analysis and visualisation. ACM recruited participants and curated clinical information of patients. MRP and ALR performed experiments and curated data. ZA-O contributed to the data analysis and revised the manuscript. LW, MAK, AA, TMM and MM provided patient and laboratory samples and reviewed the manuscript. MRZ provided funding, supervision and edited the manuscript. LC contributed to the design and methodology and revised the manuscript. ESG conceptualised developed methodology, supervised and administered the study. All authors have reviewed and approved the final manuscript.

\section{FUNDING INFORMATION}

This study was funded by an NHMRC Grant (1117911) obtained by MRZ, MM, ESG and MAK, a Cancer Council Grant (1100249) awarded to ESG, MRZ and MM 
and grants from the Department of Health Western Australia, the Spinnaker Foundation and the Perpetual Foundation to MRZ, ESG, MM and MAK. ESG is supported by a fellowship from Cancer Council WA. LW is supported by an NHMRC Postgraduate Scholarship (1190643). ACM was supported by a fellowship from the Western Australia Health Translational Network. GM was supported by a scholarship from the School of Medical and Health Sciences at Edith Cowan University.

\section{COMPETING INTERESTS}

The following authors have received travel support from MAK [Merck Sharp and Dohme (MSD), Bristol-Myers Squibb (BMS) and Merck Serono], TMM [BMS, Novartis, AstraZeneca (AZ)] and ESG [MSD]. The following authors sit on advisory boards: TMM [BMS, MSD, Novartis, AZ] and MM [BMS, AZ, Roche, MSD]. All remaining authors have declared no competing interests.

\section{ADDITIONAL INFORMATION}

Supplementary information The online version contains supplementary material available at https://doi.org/10.1038/s41416-021-01507-6.

Correspondence and requests for materials should be addressed to E.S.G.
Reprints and permission information is available at http://www.nature.com/ reprints

Publisher's note Springer Nature remains neutral with regard to jurisdictional claims in published maps and institutional affiliations.

(i) Open Access This article is licensed under a Creative Commons Attribution 4.0 International License, which permits use, sharing, adaptation, distribution and reproduction in any medium or format, as long as you give appropriate credit to the original author(s) and the source, provide a link to the Creative Commons license, and indicate if changes were made. The images or other third party material in this article are included in the article's Creative Commons license, unless indicated otherwise in a credit line to the material. If material is not included in the article's Creative Commons license and your intended use is not permitted by statutory regulation or exceeds the permitted use, you will need to obtain permission directly from the copyright holder. To view a copy of this license, visit http://creativecommons. org/licenses/by/4.0/.

(c) The Author(s) 2021 